\title{
A method for detecting and preventing negative RNA interference in preparation of lentiviral vectors for siRNA delivery
}

\author{
DEMIN ZHOU, ${ }^{1,2}$ JING ZHANG, ${ }^{2}$ CUIYING WANG, ${ }^{2}$ JOSHUA R. BLIESATH, ${ }^{2}$ QIUCHEN HE, ${ }^{2}$ DEHUA YU, ${ }^{2}$ \\ ZHANG LI-HE, ${ }^{1}$ and FLOSSIE WONG-STAAL ${ }^{2}$ \\ ${ }^{1}$ State Key Laboratory of Natural and Biomimetic Drugs, School of Pharmaceutical Sciences, Peking University, Beijing 10083, China \\ ${ }^{2}$ iTherX Inc., San Diego, California 92121, USA
}

\begin{abstract}
The lentiviral vector is a useful tool for delivery of hairpin siRNA (shRNA) into mammalian cells. However, the efficiency of this system for carrying double-stranded siRNA (dsRNA) has not been explored. In this study we cloned the two forms of siRNAcoding sequence, a palindromic DNA with a spacer loop for shRNA and a double-stranded DNA with opposing Pol III promoters for dsRNA, into lentiviral DNA vectors, and compared their viral vector production yields. Our results indicate that sharply lower titer vector was obtained for dsRNA while much higher titer vector was produced for shRNA, posing a fundamental concern whether siRNA-carrying viral RNA itself is an inherent target of RNAi. Further experimental analyses using packaging cells that either allow or do not allow siRNA transcription indicate that the shRNA-carrying viral RNA is resistant to RNAi but the viral RNA carrier for dsRNA is not, offering a linker of RNAi bias-target secondary structure that causes shRNA vector to evade RNAi degradation. More importantly, the poor yield of dsRNA vector production was restored when a novel packaging cell line was used that blocks the antisense strand from dsRNA duplexes. This method has important implications for the RNAi field, especially for those who are using lentiviral dsRNA and dsRNA libraries for various biological discovery and therapeutic interventions.
\end{abstract}

Keywords: RNAi; inducible system; lentiviral vector; siRNA packaging

\section{INTRODUCTION}

RNA interference (RNAi) is the process of using specific sequences of double-stranded RNA to knock down the expression level of sequence-homologous genes. RNAi is a ubiquitous pathway in nearly every eukaryote studied thus far and represents a unique form of post-transcriptional gene silencing (Waterhouse et al. 2001; McManus and Sharp 2002; Hannon and Rossi 2004; Meister and Tuschl 2004; Mello and Conte 2004). The real trigger of RNAi is a small double-stranded RNA that is 21-23 bases in length (Elbashir et al. 2001; Grishok et al. 2001; Hutvagner et al. 2001), which assembles with RISC in the cytoplasm (Liu et al. 2004; Song et al. 2004; Sontheimer and Carthew 2004) and initiates a complicated cascade for the ultimate

Reprint requests to: Demin Zhou, State Key Laboratory of Natural and Biomimetic Drugs, 38 Xueyuan Road, Beijing 100191, China; e-mail: deminzhou@bjmu.edu.cn; fax: 86-10-8280-5519.

Article published online ahead of print. Article and publication date are at http://www.rnajournal.org/cgi/doi/10.1261/rna.985209. degradation of sequence-homologous RNAs (Fire et al. 1998; Hammond et al. 2000). Such ability of small interfering RNA (siRNA) in mammalian cells will undoubtedly revolutionize the study of functional genomics, the discovery of drug targets, and even the treatment of human diseases (Zhou et al. 2006). The intracellular expression of siRNA can be mediated from a short palindromic sequence with an intervening loop (shRNA) (Brummelkamp et al. 2002; Sui et al. 2002; Zhou et al. 2008), or from a doublestranded DNA sequences with dual, opposing promoters (dsRNA) (Fig. 1; Tran et al. 2003; Zheng et al. 2004; Ke et al. 2006). We have recently shown that expression of short double-stranded RNA from opposing promoters can be used efficiently to generate partially randomized siRNA libraries targeting conserved gene families (Yang et al. 2006).

Retroviral gene transfer is a powerful technique for stable introduction of heritable genetic material into mammalian cells (Mulligan 1993). Retroviruses pseudotyped with the VSV-G protein efficiently infect most cell types, and the resultant provirus integrates into the host chromosome for 
A

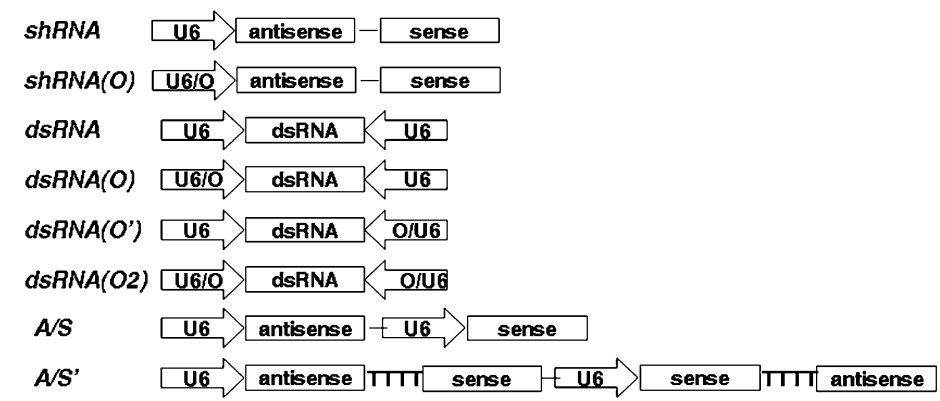

B

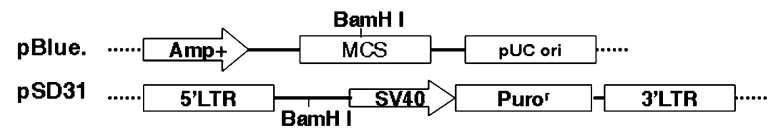

FIGURE 1. (A) Schematic representation of a variety of siRNA expression cassettes used in this study: shRNA, a shRNA expression cassette with a single mouse U6 promoter for transcription of the palindrome DNA template; $\operatorname{sh} R N A(O)$, a shRNA expression cassette with an inducible mouse U6 promoter wherein a TetO sequence was inserted between TATA box and PSE for conditional shRNA expression (Zhang et al. 2007); $d s R N A$, a dsRNA expression cassette with dual opposing mouse U6 promoters for simultaneous transcription of the sense strand and antisense strand in convergent manner (Tran et al. 2003; Zheng et al. 2004); dsRNA(O), a dsRNA expression cassette with the antisense strand expression driven by the inducible U6 promoters (Zhang et al. 2007); $d s R N A\left(O^{\prime}\right)$, a dsRNA expression cassette with the sense strand expression driven by the inducible U6 promoters; $d s R N A(\mathrm{O} 2)$, a dsRNA expression cassette with both strand expressions driven by the inducible U6 promoter; $A / S$, an alternative dsRNA expression cassette with tandem U6 promoters driving antisense and sense strands expressions (Lee et al. 2002; Miyagishi and Taira 2002). (B) Two DNA vectors used in this study for cloning siRNA expression cassettes at the BamH I site: pBlue, the commercial pBlueScript DNA vector from Strategene; pSD31, a pHIV-7 derived lentivirus vector with a BamH I site downstream from the $5^{\prime}$ LTR for cloning siRNA expression cassettes (Zhang et al. 2007).

stable gene expression (Yee 1999; Buchschacher and WongStaal 2000). In addition, the use of lentiviral vectors further broadens the host range to include nondividing and terminally differentiated cells (Quinonez and Sutton 2002; Zhou et al. 2006). Lentiviral vectors that stably introduce RNAi into mammalian cells can be used to achieve longterm silencing of various human genes (Qin et al. 2003; Tiscornia et al. 2003). However, delivery of silencing siRNA by lentiviral vectors may be more problematic than delivery of regular genes.

A fundamental concern is whether siRNA-carrying viral RNA itself is an inherent target of RNAi during the packaging process. The production of siRNA lentiviral vectors is generally by transient co-transfection of the siRNA-encoding lentiviral DNA vector with packaging plasmids that encode the retroviral structural genes and essential regulatory genes into cells (Pear 1996; Yee 1999). In these packaging cells, the full-length viral RNA embedded with the siRNA sequence is transcribed via a polymerase II promoter initiated at the $5^{\prime}$ LTR and terminated at the $3^{\prime}$ LTR. Concomitantly, transcription of siRNA is initiated at the internal Pol III promoter(s). The sequence homology of the siRNA to the siRNA-carrying viral RNA will, in theory, elicit RNAi against the viral vector RNA itself and thus can potentially reduce the viral RNA levels, and subsequently viral vector titers.

Here we developed a method aimed at detecting the potential RNA interference in siRNA-carrying viral vector production. Our experimental results not only disclose an RNAi target bias in preparations of shRNA and dsRNA vectors, but also offer a clear linker of RNAi biasRNA target secondary structures. More importantly, this method provides an approach that prevents the negative RNAi and thus restores the yield of dsRNA vector production. These results have important implications for RNAi field especially those who are using the lentiviral dsRNA and dsRNA library for various biological discovery and therapeutic interventions.

\section{RESULTS AND DISCUSSION}

\section{Extremely poor efficiency of lentiviral vectors for delivery of dsRNA library}

We have previously demonstrated a method to generate a partially randomized dsRNA library targeting nuclear hormone receptor gene families (Yang et al. 2006). In order to test whether this library can be delivered efficiently by lentiviral vectors, we cloned their DNA templates into a hU6-dsRNA-mU6 expression cassette (Fig. 1; Yang et al. 2006), and ligated into a lentiviral DNA vector, pSD31 (Zhang et al. 2007; Zhou et al. 2008). The yield of RNA vector produced from a popular packaging cell line, 293FT, was tittered. As shown in Figure 2, extremely low titer of dsRNA library vector was obtained, contrasting sharply with the titer of pSD31 backbone only. The poor yield of RNA vector production for this dsRNA library poses a general concern whether the lentiviral RNA vector is a good tool for delivery of silencing siRNA.

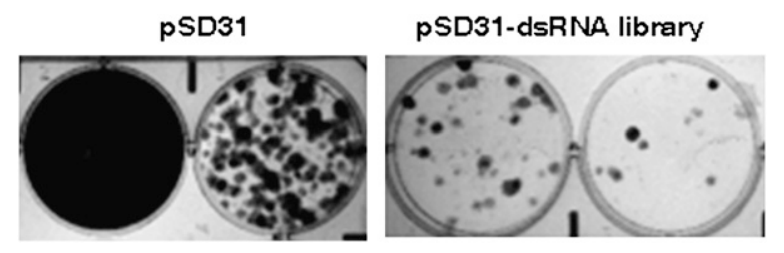

FIGURE 2. Extremely low efficiency of a lentiviral vector for delivery of a dsRNA library. The vector stocks were diluted 3000- and 1000fold for tittering. pSD31 is the lentiviral backbone vector. 


\section{Potential RNA interference in preparation of a siRNA-carrying lentiviral vector}

In order to figure out this puzzle, we chose a wellcharacterized p53-targeting siRNA and cloned two forms of its coding sequence (shRNA versus dsRNA) into pSD31 (Fig. 1). We define sense and antisense strand base on target p53 mRNA. The convergent cassette can be placed in two orientations in pSD31. We chose only one orientation for further discussion, i.e., the sense strand is sequence identical to the simultaneously transcribed vector RNA, so that both p53 mRNA and viral RNA are dual targets of the same RNAi. The mouse U6 promoter (mU6) in dsRNA expression cassette is changed to human U6 promoter (hU6) to avoid sense and antisense transcription nonequivalence from different U6 promoters. The shRNA has been delivered into mammalian cells via a lentiviral vector and displayed robust p53 knockdown in stable transduction (Zhang et al. 2007). In order to test whether dsRNA can be delivered in the same way as shRNA, we packaged the plasmids pSD31-dsRNA and pSD31-shRNA to produce siRNA lentiviral vectors. High titer vectors were obtained for pSD31-shRNA $\left(\sim 1 \times 10^{6} / \mathrm{mL}\right)$, comparable to the backbone vector pSD31, while $>30$-fold lower titers were obtained for pSD31-dsRNA (Fig. 3A).

To test whether siRNA is active in the process of lentivector production and explore the molecular basis of

A
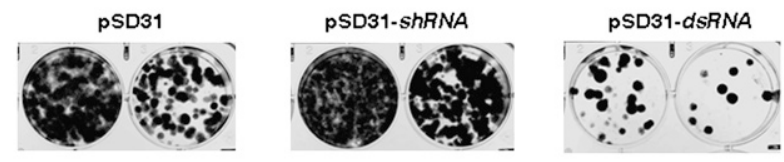

B

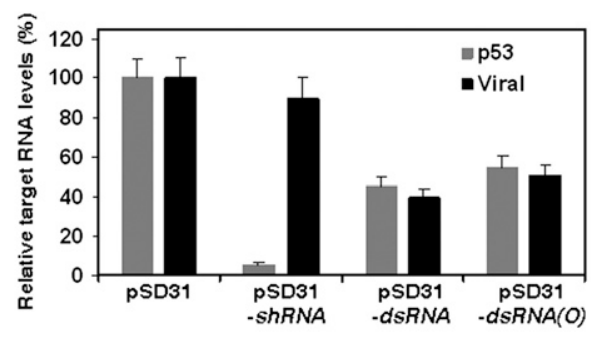

C

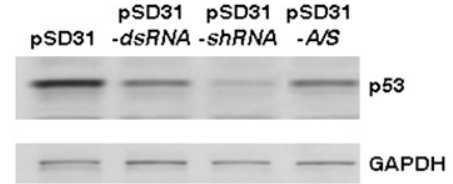

FIGURE 3. (A) Comparison of viral vector productions from 293FT cells transfected with pSD31, pSD31-shRNA, pSD31-dsRNA, or pSD31-A/S. The vector stocks were diluted 3000and 1000-fold for tittering. (B) Taqman analyses of p53 and viral RNA knockdown in 293FT cells transfected with pSD31-shRNA, pSD31-dsRNA, or pSD31-A/S. The data were relative to the levels of p53 mRNA and viral RNA in pSD31-transfected 293FT cells. Data shown are from an experiment conducted in triplicate for each sample. (C) Western analyses of p53 knockdown in 293FT cells transfected with pSD31-shRNA, pSD31-dsRNA, or pSD31-A/S.

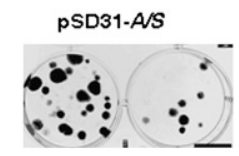

titer variation, we measured the levels of viral RNA and p53 mRNA, two potential targets of the same RNAi, in the packaged 293FT cells. The expression levels of p53 mRNA and viral RNA in pSD31-transfected 293FT cells were used as controls. We found that $\sim 10 \%$ of p53 mRNA and almost $90 \%$ of viral RNA were detected in pSD31-shRNAtransfected cells, in significant contrast to pSD31-dsRNAtransfected cells, wherein both targets were equally reduced $\sim 60 \%$ (Fig. 3B). Clearly, the endogenous p53 mRNA was knocked down by both siRNAs and further knockdown was achieved for shRNA compared with dsRNA, which was also confirmed in Western blotting analysis (Fig. 3C). These data suggest that in the packaging process the shRNA is very efficient but just preferentially degrades p53 mRNA not the viral RNA while the dsRNA, despite its relatively lower efficiency, knocks down both targets to the same extent. These results raise a question whether siRNAcarrying viral RNA itself is an inherent target of siRNA during the packaging process.

\section{The effect of RNAi blocking on siRNA-carrying lentiviral vector productions}

To answer this fundamental question we created a special packaging cell line wherein siRNA expression is capable of being regulated. This novel cell line, 293FTR, was generated by stable transduction of 293FT cells with lentiviral vector pSD400, which overexpresses the tetracycline repressor (TetR) in transduced cells (Zhang et al. 2007; He et al. 2008). The expression level of TetR in 293FTR cells is shown in Figure 4, A and B. As compared with T-Rex, a commercial TetR-expressing cell line from Invitrogen, the TetR mRNA level in 293FTR is $\sim 15$-fold higher based on Taqman analysis and the protein level is further greater based on Western blotting. We then transfected 293FTR cells with pSD31-shRNA and pSD31-shRNA(O). In the latter construct the shRNA expression was driven by a TetO-tethered inducible U6 promoter (Zhang et al. 2007), and therefore should be suppressed in 293FTR cells. As shown in Figure $4 \mathrm{C}$, there is no detectable p53 knockdown in pSD31-shRNA(O)-transfected 293FTR cells based on Taqman data, in contrast to $80 \%$ knockdown by the same plasmid in 293FT cells and 90\% knockdown in both pSD31-shRNAtransfected 293FT and 293FTR cells; this indicates that the inducible U6 promoter for siRNA expression was completely inactivated in 293FTR cells. 
A

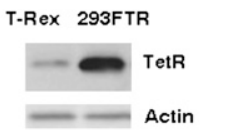

B

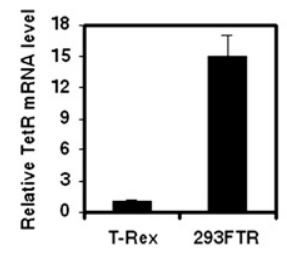

$\mathrm{C}$

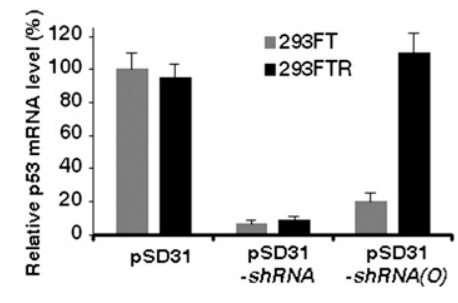

FIGURE 4. (A) Analyses of TetR overexpression in 293FTR cells by Western blotting. Actin is an internal marker and T-Rex is a control cell line purchased from Invitrogen that stably expresses TetR. (B) Taqman confirmation of TetR overexpression in 293FTR cells. The TetR mRNA level in T-Rex is normalized to 1. Both samples are tested in triplicate. $(C)$ Taqman analyses of p53 knockdown in 293FT and 293FTR cells transfected with pSD31-shRNA and pSD31$\operatorname{shRNA}(O)$. The data were relative to p53 mRNA level in pSD31transfected 293FT cells. Data are derived from an experiment conducted in triplicate.

Next we compared the efficiency of viral vector productions from the developed 293FTR cell line and its parental 293FT cell line. As shown in Figure 5A, pSD31, pSD31shRNA, and pSD31-dsRNA yielded the same titer in both cells $\left(\sim 1 \times 10^{6}\right.$ for pSD31-shRNA and $\sim 3 \times 10^{4}$ for pSD31-dsRNA), indicating that TetR expression itself in 293FTR had no negative effect on viral RNA transcription and packaging. We then used these two packaging cell lines to validate the differential RNAi susceptibility to shRNA and dsRNA viral vectors. The titer for packaging pSD31$\operatorname{shRNA}(O)$ from 293FTR cells was comparable to that from 293FT cells, indicating shRNA expression or not has no effect on shRNA vector production. In contrast, the titer of pSD31-dsRNA $(O)$, whose antisense strand expression was driven by the inducible U6 promoter, from 293FTR cells was dramatically increased $\left(\sim 1 \times 10^{6}\right)$ (Fig. 5A), indicating dsRNA expression significantly interferes with viral RNA level. Taqman analyses of viral RNA and p53 mRNA in transfected 293FTR cells indicated neither was significantly knocked down by pSD31-shRNA(O) or pSD31-dsRNA(O) (Fig. 5B), confirming the effectiveness of TetR on suppressing siRNA expression. Considering the combined results from 293FTR and 293FT cells, one can conclude that the viral vector titer directly correlated with the level of the viral vector RNA, and that shRNA, but not dsRNA, exhibited a target bias against the corresponding viral RNA versus p53 mRNA. We also compared pSD31-dsRNA with pSD31-A/S (Fig. 1A), an alternative dsRNA expression system with tandem promoters (Lee et al. 2002; Miyagishi and Taira 2002), on viral vector production and p53 and viral RNA gene knockdown. Their identical behavior (Fig. $3 \mathrm{~A}-\mathrm{C}$ ) excluded potential promoter interference between opposing promoters that may cause RNAi variation on vector degradation.

\section{Mechanism underlying RNAi bias in preparations of ShRNA and dsRNA viral vectors}

The RNAi bias in preferential degradation of the dsRNA viral vector rather than the shRNA vector could be due to either the target RNA or siRNA effector molecule, or both. To distinguish these possibilities, we co-transfected pSD31shRNA(O) and pBlue dsRNA (Fig. 1) into 293FTR cells, wherein the expression of shRNA, but not the dsRNA, was blocked, and measured the viral RNA level relative to that in pSD31/pBlue-transfected-293FTR cells. As shown in Figure 5C, there was no viral RNA knockdown either, indicating the shRNA-carrying viral RNA is resistant also to dsRNA. In a reciprocal experiment, we co-transfected pSD31-dsRNA(O) and pBlue-shRNA (Fig. 1) into 293FTR cells, wherein the dsRNA expression was blocked but not the shRNA. Taqman data demonstrated that $>70 \%$ of viral RNA was degraded (Fig. 5C), indicating dsRNA-delivering viral RNA is also a good target for the shRNA. Therefore, shRNA-carrying viral RNA, but not dsRNA-encoding viral RNA, was resistant to RNAi.

Many factors may potentially attenuate RNAi efficacy. There have been conflicting reports on whether local secondary structures of target RNA restrict the accessibility of RISC and decrease siRNA efficacy (Harborth et al. 2001; Bohula et al. 2003; Ding and Lawrence 2003; KretschmerKazemi and Sczakiel 2003; Vickers et al. 2003; Wunsche and Sczakiel 2005; Pei and Tuschl 2006). It has been difficult to assess the target accessibility based on a reliable prediction of target secondary structure, since other factors, such as RNA-protein interactions, may potentially affect target accessibility to RNAi. Our observation of differential susceptibility of sh- and ds-viral RNA to RNAi degradation may provide some clarification on this controversy. The shRNA-vector genome contains a palindromic sequence encoding the shRNA, as opposed to the dsRNA-vector genome, which contains a flat sequence encoding the antisense and sense strands (Fig. 1A). Such a difference leads to significant structural variation at the RNAi target position: a hairpin stem for the shRNA-carrying viral RNA versus a nonstructured flattened sequence for the dsRNAencoding viral RNA, with the remaining part of the two viral genomic transcripts being the same (Fig. 5D). Cotransfection experiments demonstrated that the viral RNA with a hairpin stem is resistant to both shRNA and dsRNA (Fig. 5C). In contrast, the flat ds-viral RNA containing the sense strand sequence is sensitive to both siRNAs (Fig. $5 \mathrm{C}, \mathrm{D})$. Our results would strongly suggest that it is the local secondary structures within the target viral RNA that determines vector sensitivity to RNAi degradation. We conclude that local hairpin secondary structures in the target viral RNA do strongly affect RNAi efficacy, possibly preventing siRNA accessory, and thus make it resistant to RNAi. This is consistent with the very recent reports that the lentiviral vector can escape from RNAi by evolving an 
A

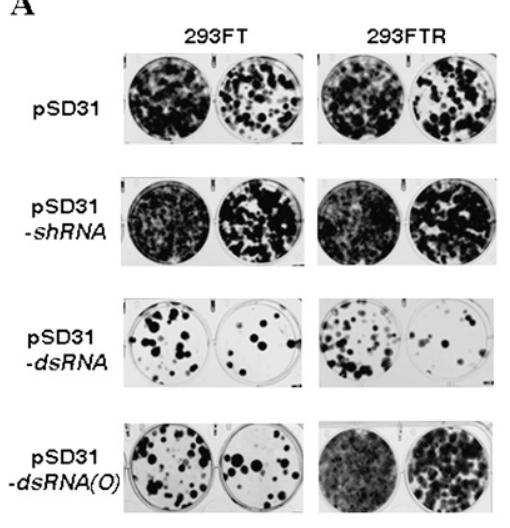

B
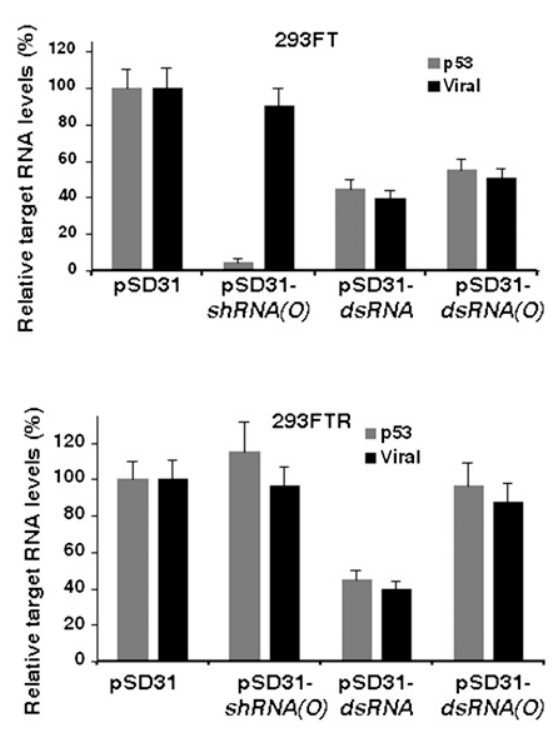

$\mathrm{C}$

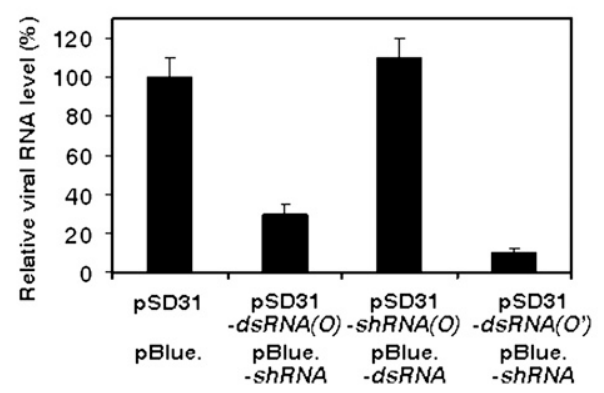

FIGURE 5. (A) Comparisons of viral vector productions from 293FT or 293FTR cells transfected with pSD31, pSD31-shRNA, pSD31-dsRNA, or pSD31-dsRNA(O). The vector stocks were diluted 3000- and 1000-fold for tittering. (B) Taqman analyses of p53 and viral RNA knockdown in above-transfected 293FT cells and 293FTR cells. The data were relative to the levels of p53 mRNA and viral RNA in pSD31-transfected 293FT cells. All samples were tested in triplicate. $(C)$ Taqman analyses of viral RNA knockdown in 293FTR cells which were co-transfected with pSD31-dsRNA(O)/pBlue-shRNA, pSD31-shRNA(O)/pBlue- $d s R N A$, or pSD31-dsRNA $\left(O^{\prime}\right) / \mathrm{pBlue}-s h R N A$. The data were relative to the viral RNA level in pSD31/ pBlue co-transfected 293FTR cells. All samples were tested in triplicate. $(D)$ Conclusive analyses of RNAi bias toward shRNA- and dsRNA-carrying viral RNAs. The shRNA-carrying viral RNA is resistant to both shRNA and dsRNA (dotted arrow line) while the dsRNAcarrying viral RNA is sensitive to both siRNAs (solid arrow line). Obviously, the hairpin structure within the shRNA-carrying viral RNA significantly affects RNAi efficacy.

The relative contribution of dsRNA duplexes on dsRNA vector degradation

Since dsRNA vector is sensitive to concomitantly expressed dsRNA, we tried to use different dsRNA expression cassettes (Fig. 1A) to evaluate the relative contribution of dsRNA duplexes on viral RNA degradation. As shown in Figures 5A and $7 \mathrm{~A}$, transfection of pSD31-dsRNA(O) into 293FTR cells with the expression of the antisense strand being locked significantly improved the vector production with maximum titer obtained $\left(\sim 1 \times 10^{6} \mathrm{cfu} / \mathrm{mL}\right)$, indicating siRNA becomes inactive in the absence of the antisense strand. This is confirmed by direct analysis of the viral RNA levels (Fig. 5B). These results support the idea that the antisense strand is the main effective component in RNAi, and blocking its expression completely restores viral RNA vector production. In a parallel experiment, we evaluated the role of the sense strand by transfection of pSD31-dsRNA $\left(O^{\prime}\right)$ into 293FTR and 293FT cells. Interestingly, blocking the sense strand expression in 293FTR cells did not enhance vector production as compared to pSD31-dsRNA (Fig. 7A), and did not reverse viral RNA degradation (Fig. 7B), indicating that the sense strand is likely dispensable for RNAimediated vector deletion.

One potential cause of titer decrease in packaging pSD31- $d s R N A\left(O^{\prime}\right)$ using $293 \mathrm{FT}$ or 293FTR cells may originate from impairment of the viral RNA transcription or packaging due to the inserted TetO sequence in the DNA template. We packaged pSD31-dsRNA(O2), which contains two TetO sequences in the

alternative structure in its RNA genome (ter Brake and Berkhout 2007; Westerhout and Berkhout 2007).

The role of viral RNA structure was further demonstrated by modification of construct A/S into A/S' (Fig. 1A), using tandem Pol III cassettes terminated by T5 as a spacer followed by antisense sequence in the vector. Both $\mathrm{A} / \mathrm{S}^{\prime}$ and $\mathrm{A} / \mathrm{S}$ should produce the same dsRNA in transfected cells with the later one mimicking shRNA-carrying viral RNAs. As expected, the RNA level of $A / S^{\prime}$ and its titer were much higher than that of A/S (Fig. 6A,B), strongly supporting that the susceptibility of A/S (low titer) to RNAi is due to lack of RNA secondary structure.
DNA template (Fig. 1A), and found that its viral titer, $4 \times$ $10^{4} \mathrm{c} / \mathrm{mL}$, is even a little higher than that from packaging pSD31-dsRNA (Fig. 7A), excluding the possibility of TetO disturbance on viral vector production. In addition, similar to packaging pSD31-dsRNA(O), maximum titer was obtained for packaging pSD31-dsRNA(O2) using the novel 293FTR packaging cell line, suggesting that blocking both strands of dsRNA or just the sense strand can inactivate RNAi and thus significantly improve dsRNA vector production.

It is well known that siRNA is a 21-23-base-pair doublestranded RNA that triggers RNAi via the aid of the RISC. However, recent biochemical studies revealed that siRNA 
A

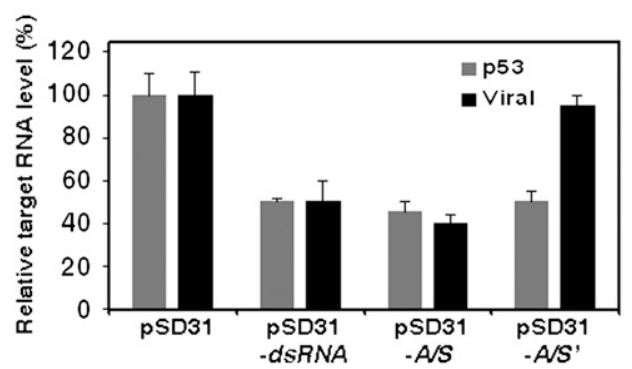

B

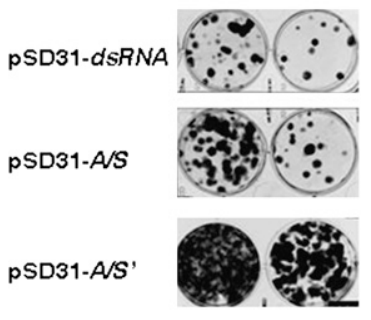

lentiviral vectors for delivery of the dsRNA library, we cloned the semi-randomized nuclear hormone receptor dsRNA library (Yang et al. 2006) into the $d s R N A(O)$ expression cassette and then ligated it into pSD31. The $d s R N A(O)$ expression cassette allows conditional repression of dsRNA (antisense strand) expression during vector production. As a result, the titer of dsRNA vectors was dramatically enhanced and such enhancement was reversed when $100 \mathrm{ng} / \mathrm{mL}$ of doxycycline was added to the 293FTR cell culture that induces dsRNA expression (Fig. 8). In a parallel experiment using 293FT as packaging cells, lower titer of dsRNA vectors was obtained either in

duplexes exhibit strand asymmetry on RISC assembling, and proposed a concept of single-stranded siRNA and double-stranded siRNA (Martinez et al. 2002; Holen et al. 2003; Khvorova et al. 2003; Schwarz et al. 2003). Here we utilized the novel 293FTR cell line to selectively block the expression of either strand of the dsRNA duplexes, and confirmed that the antisense strand is the main effective component of RNAi (Martinez et al. 2002; Holen et al. 2003; Khvorova et al. 2003; Schwarz et al. 2003), since inhibiting its transcription in the pSD31- $d s R N A(O)$ vector completely restored vector production (Figs. 5,7 ). On the contrary, blocking the sense strand in the pSD31-dsRNA $\left(O^{\prime}\right)$ vector had almost no positive effect on vector production (Fig. 7). These results support the concept of single-stranded antisense siRNA and double-stranded siRNA, i.e., both are effective on gene silencing (Martinez et al. 2002; Holen et al. 2003; Khvorova et al. 2003; Schwarz et al. 2003). However, unlike previous studies which indicated that the singlestranded siRNA is $\sim 5-10$ folds less effective than double-stranded siRNA, we found that they displayed an identical effect on viral RNA degradation (Fig. $7 A, B)$. Our study differs from others in that we have constitutive expression of the antisense strand within intact cells versus transient presence in cell lysates. Therefore, the low potency of the singlestranded siRNA previously observed may not reflect its real activity, but rather its lower stability compared with doublestranded RNA (Holen et al. 2003).

\section{Preparation of high titer of lentiviral vectors for delivery of dsRNA libraries}

To test whether the 293FTR cell line can be used for preparation of high titer of the presence of the inducer or not. Therefore, 293FTR is a unique packaging cell line for preparation of high titer of lentiviral vectors for delivery of dsRNA libraries.

To our knowledge, so far only shRNA has been reported to deliver by retroviral vectors for stable transduction. Here, our novel packaging system provides a mechanistic insight into lack of efficiency for dsRNA vector production. And also, as a proof of concept, we have demonstrated the powerful utility of this system for efficient delivery of the partial-randomized dsRNA library targeting specific gene family, which could be constructed with relative ease (Yang et al. 2006). It can be envisioned that other combinatorial siRNA libraries based on dsRNA may also be constructed with less technical challenge than those based on shRNA. The availability of this novel packaging system we describe would allow stable transduction of such dsRNA libraries, which should have important implications for the RNAi field, especially for those who are using lentiviral dsRNA
A

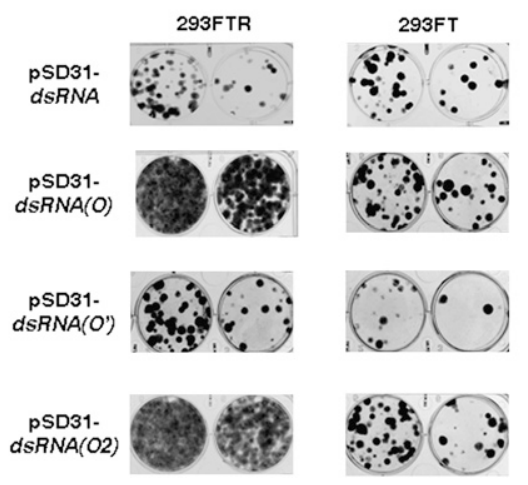

B

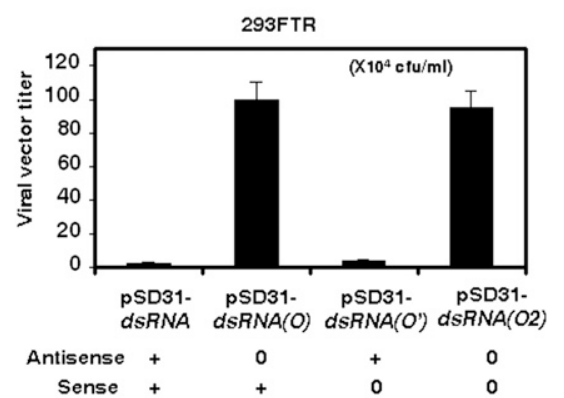

FIGURE 7. (A) Comparisons of viral vector productions from 293FT or 293FTR cells transfected with pSD31-dsRNA, pSD31-dsRNA(O), pSD31-dsRNA(O), or pSD31-dsRNA(O2). The vector stocks were diluted 3000- and 1000-fold for tittering. (B) Comparative analyses of blocking or reducing siRNA duplexes on viral vector productions, which reversely reflects RNAi. The regular expression of the sense or antisense strand was marked by "+"; blocking expression was marked as " 0 ". All viral vectors were tittered in triplicate. 


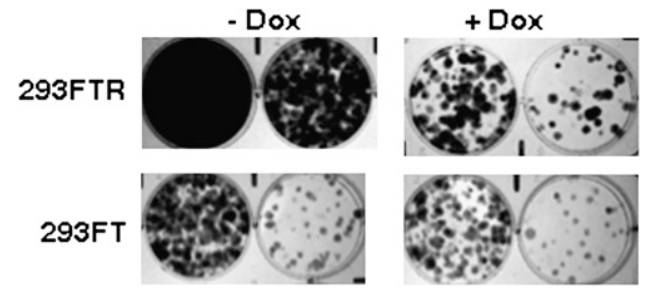

FIGURE 8. Comparisons of viral vector productions from 293FTR or 293FT cells transfected with the pSD31-dsRNA(O) library in the presence of $(+$ Dox $)$ or absence of (- Dox) doxycycline.

and dsRNA libraries for various biological discovery and therapeutic interventions.

\section{MATERIALS AND METHODS}

\section{Construction of various shRNA and dsRNA expression systems}

shRNA, a p53-targeting hairpin siRNA expression containing a mouse U6 promoter, and $\operatorname{sh} R A(O)$, the corresponding inducible cassette with a TetO sequence inserted into the mU6 promoter between the TATA box and PSE region (Fig. 1A), were constructed by routine PCR as described previously (Zhang et al. 2007). dsRNA and $A / S$, double-stranded siRNA expression cassettes with dual mouse U6 promoters driving the siRNA sense and antisense strand expression in a convergent orientation $(d s R N A)$ or in tandem $(A / S)$, were constructed by PCR and PCR ligation. The same method was used to constructed $d s R N A(O)$, dsRNA $\left(O^{\prime}\right)$, and $d s R N A(O 2)$, three inducible cassettes of dsRNA with TetO sequence inserted into one or two of U6 promoters between the TATA box and PSE for respectively adjusting the expression of antisense, sense, and both strands. The TetO sequences and the sense strand of siRNA were 5'-CTCTATCATTGATAGAGT-3' and $5^{\prime}$-GACTCCAGTGGTAATCTAC-3'. pBlueScript (pBlue-) vector was purchased from Stratagene for siRNA-encoding DNA transient transfection. pSD31 was modified from pHIV-7 vector for siRNA packaging (Fig. 1B; Zhang et al. 2007).

\section{Cell culture, transient transfection, and lentiplasmid packaging}

Mammalian cells used in this study including 293FT and its derivate 293FTR were cultured in Dulbecco's modified Eagle's medium supplemented with $10 \%$ fetal calf serum. Experiments for lentiviral vector production using $293 \mathrm{FT}$ or $293 \mathrm{FTR}$ as a packaging cell line was performed according to Invitrogen's standard protocols. All packaging and following tittering experiments except the dsRNA library were carried out in parallels-exactly under the same conditions to exclude other potential factor variations that may attenuate viral vector titter. Briefly, subconfluent $293 \mathrm{FT}$ or 293FTR packaging cells in a T75 flask were co-transfected (afterward referred to as transfection if not specially noted) with $20 \mathrm{mg}$ of a recombinant lenti-plasmid (pSD31shRNA, pSD31-dsRNA, pSD31-shRNA(O), pSD31-dsRNA(O), pSD31-dsRNA $\left(O^{\prime}\right)$, pSD31-dsRNA(O2), or pSD31), $15 \mathrm{mg}$ of pCMV-DR8.91, and $5 \mathrm{mg}$ of pMD2G-VSVG by calcium phos- phate precipitation. The medium was changed in $6 \mathrm{~h}$ and lentiviral vectors were harvested at $72 \mathrm{~h}$. The cell pellets were washed once with PBS buffer and total RNA was extracted for Taqman analysis.

\section{Creation of a novel 293FTR package cell line}

A 293FTR cell line was created by stable transduction of 293FT with pSD400 (Zhang et al. 2007) as follows: $1 \times 10^{6}$ of 293FT cells were seeded in a T25 flask and transduced with pSD400 at day 2 in the presence of $8 \mathrm{mg} / \mathrm{mL}$ polybrene. Selection was performed from day 3 by $450 \mathrm{ng} / \mathrm{mL}$ puromycin until parental cells from a parallel experiment completely died.

\section{Western blot analysis}

Cells were washed once with PBS buffer and lysed in boiling lysis buffer (1\% SDS, $50 \mathrm{mM}$ Tris at $\mathrm{pH} 7.4,0.15 \mathrm{M} \mathrm{NaCl}, 1 \mathrm{mM} \mathrm{NaF}$, $10 \mathrm{mM}$ phenylmethylsulfonyl fluoride, $1 \mathrm{mM}$ sodium orthovanadate, $1 \mathrm{mM}$ EDTA) for $5 \mathrm{~min}$ and passed through a 27-gauge needle. Lysates were cleared by centrifugation at $12,000 \mathrm{~g}$ for $1 \mathrm{~min}$, and protein concentration was determined using a BioRad DC protein assay. Equal amounts of protein were separated by $4 \%-20 \%$ SDS-PAGE before transfer to nitrocellulose membranes. Membranes were blocked with $5 \%$ skim milk or $3 \%$ bovine serum albumin in TBST $(10 \mathrm{mM}$ Tris at pH 7.5, $150 \mathrm{mM}$ $\mathrm{NaCl}, 0.1 \%$ Tween 20 ) for $1 \mathrm{~h}$ at room temperature. Primary and secondary antibodies were used according to the manufacturer's instructions, followed by detection with enhanced chemiluminescence technique (Amersham Biosciences).

\section{Taqman analysis of the RNA level of endogenous p53 and exogenous viral RNA in the packaged 293FT and 293FTR cells}

Quantitative real-time RT-PCR (TaqMan assay) was carried out to determine the expression level of endogenous p53 mRNA and viral RNA in 293FT and 293FTR cells that were transfected with variant pSD31-recombinant plasmid and structural plasmids. Dual-labeled fluorogenic probes and primers were synthesized at Integrated DNA Technologies, Inc. and a one-step real-time RTPCR was carried out using an ABI PRISM 7700 sequence detection system (Applied Biosystems) as described previously (Hu et al. 2004). Real-time PCR data were collected using the ABI PRISM 7700 sequence detection system. Relative quantification of the p53 mRNAs and viral RNA were achieved according to the principle of the standard-based quantitative PCR method as described previously ( $\mathrm{Hu}$ et al. 2004). The error bar for triplication of each sample is $\sim 15 \%$.

\section{Titration of the lentiviral particles}

According to Invitrogen's protocol, $1 \times 10^{5} \mathrm{HT} 1080$ cells were plated in each well of a six-well plate. On the following day, two parallels of each viral stock diluted at 3000- and 1000-fold were used to transduce the cells in the presence of polybrene $(8 \mathrm{mg} /$ $\mathrm{mL}$ ). After $24 \mathrm{~h}$ the medium was changed to fresh medium supplied with $450 \mathrm{ng} / \mathrm{mL}$ puromycin for selection. The medium was changed every three days until day 10 when the medium was replaced with $2 \mathrm{~mL}$ of Coomassie blue stain and incubated for $30 \mathrm{~min}$. 
After aspirating the stain and gently washing by water, the number of colonies present in each well was counted to get the average titer.

\section{ACKOWLEDGMENTS}

We thank Dr. Lianxin Zheng (Novartis) for critically reading the manuscript. This research was partly supported by National Natural Science Foundation of China (20852001), “985” Project Foundation (985-2-126-121), and The Key Laboratory Grant (20080104).

Received January 2, 2007; accepted January 7, 2009.

\section{REFERENCES}

Bohula, E.A., Salisbury, A.J., Sohail, M., Playford, M.P., Riedemann, J., Southern, E.M., and Macaulay, V.M. 2003. The efficacy of small interfering RNAs targeted to the type 1 insulinlike growth factor receptor (IGF1R) is influenced by secondary structure in the IGF1R transcript. J. Biol. Chem. 278: 15991-15997.

Brummelkamp, T.R., Bernards, R., and Agami, R. 2002. A system for stable expression of short interfering RNAs in mammalian cells. Science 296: 550-553.

Buchschacher, G.L. and Wong-Staal, F. 2000. Development of lentiviral vectors for gene therapy for human diseases. Blood 95: 2499-2504.

Ding, Y. and Lawrence, C.E. 2003. A statistical sampling algorithm for RNA secondary structure prediction. Nucleic Acids Res. 31: 7280-7301.

Elbashir, S.M., Lendeckel, W., and Tuschl, T. 2001. RNA interference is mediated by 21- and 22-nucleotide RNAs. Genes \& Dev. 15: $188-200$.

Fire, A., Xu, S., Montgomery, M.K., Kostas, S.A., Driver, S.E., and Mello, C.C. 1998. Potent and specific genetic interference by double-stranded RNA in Caenorhabditis elegans. Nature 391: 806-811.

Grishok, A., Pasquinelli, A.E., Conte, D., Li, N., Parrish, S., Ha, I., Baillie, D.L., Fire, A., Ruvkun, G., and Mello, C.C. 2001. Genes and mechanisms related to RNA interference regulate expression of the small temporal RNAs that control C. elegans developmental timing. Cell 106: 23-34.

Hammond, S.M., Bernstein, E., Beach, D., and Hannon, G.J. 2000. An RNA-directed nuclease mediates post-transcriptional gene silencing in Drosophila cells. Nature 404: 293-296.

Hannon, G.J. and Rossi, J.J. 2004. Unlocking the potential of the human genome with RNA interference. Nature 431: 371-378.

Harborth, J., Elbashir, S.M., Bechert, K., Tuschl, T., and Weber, K. 2001. Identification of essential genes in cultured mammalian cells using small interfering RNAs. J. Cell Sci. 114: 4557-4565.

He, Q.S., Tang, H., Zhang, J., Truong, K., Wong-Staal, F., and Zhou, D. 2008. Comparisons of RNAi approaches for validation of human RNA helicase A as an essential factor in hepatitis $\mathrm{C}$ virus replication. J. Virol. Methods 154: 216-219.

Holen, T., Amarzguioui, M., Babaie, E., and Prydz, H. 2003. Similar behaviors of single-strand and double-strand siRNAs suggests they act through a common RNAi pathway. Nucleic Acids Res. 31: 2401-2407.

Hu, X., Hipolito, S., Lynn, R., Abraham, V., Ramos, S., and WongStaal, F. 2004. Relative gene-silencing efficiencies of small interfering RNAs targeting sense and antisense transcripts from the same genetic locus. Nucleic Acids Res. 32: 4609-4617.

Hutvagner, G., McLachlan, J., Pasquinelli, A.E., Balint, E., Tuschl, T., and Zamore, P.D. 2001. A cellular function for the RNAinterference enzyme Dicer in the maturation of the let-7 small temporal RNA. Science 293: 834-838.

Ke, N., Zhou, D., Chatterton, J.E., Liu, G.H., Chionis, J., Zhang, J., Tsugawa, L., Lynn, R., Yu, D.H., Meyhack, B., et al. 2006. A new inducible RNAi xenograft model for assessing the staged tumor response to mTOR silencing. Exp. Cell Res. 312: 2726-2734.

Khvorova, A., Reynolds, A., and Jayasena, S.D. 2003. Functional siRNAs and miRNAs exhibit strand bias. Cell 115: 209-216.

Kretschmer-Kazemi, F.R. and Sczakiel, G. 2003. The activity of siRNA in mammalian cells is related to structural target accessibility: A comparison with antisense oligonucleotides. Nucleic Acids Res. 31: $4417-4424$.

Lee, N.A., Dohjima, T., Bauer, G., Li, H., Li, M.J., Ehsani, A., Salvaterra, P., and Rossi, J. 2002. Expression of small interfering RNAs targeted against HIV-1 rev transcripts in human cells. Nat. Biotechnol. 20: 500-505.

Liu, J., Carmell, M.A., Rivas, F.V., Marsden, C.G., Thomson, M., Song, J., Hammond, S.M., Joshua-Tor, L., and Hannon, G.J. 2004. Argonaute2 is the catalytic engine of mammalian RNAi. Science 305: 1437-1441.

Martinez, J., Patkaniowska, A., Urlaub, H., Luhrmann, R., and Tuschl, T. 2002. Single-stranded antisense siRNAs guide target RNA cleavage in RNAi. Cell 110: 563-574.

McManus, M.T. and Sharp, P.A. 2002. Gene silencing in mammals by small interfering RNAs. Nat. Rev. Genet. 3: 737-747.

Meister, G. and Tuschl, T. 2004. Mechanisms of gene silencing by double-stranded RNA. Nature 431: 343-349.

Mello, C.C. and Conte Jr., D. 2004. Revealing the world of RNA interference. Nature 431: 338-342.

Miyagishi, M. and Taira, K. 2002. U6 promoter-driven siRNAs with four uridine $3^{\prime}$ overhangs efficiently suppress targeted gene expression in mammalian cells. Nat. Biotechnol. 20: 497-500.

Mulligan, R.C. 1993. The basic science of gene therapy. Science 260: 926-932.

Pear, W. 1996. Transient transfection methods for preparation of hightiter retroviral supernatants. In Current protocols in molecular biology (eds. E. Ausubel et al.), pp. 9.11.1-9.11.18. Wiley, New York.

Pei, Y. and Tuschl, T. 2006. On the art of identifying effective and specific siRNAs. Nat. Methods 3: 670-676.

Qin, X.F., An, D.S., Chen, I.S., and Baltimore, D. 2003. Inhibiting HIV-1 infection in human T cells by lentiviral-mediated delivery of small interfering RNA against CCR5. Proc. Natl. Acad. Sci. 100: $183-188$.

Quinonez, R. and Sutton, R.E. 2002. Lentiviral vectors for gene delivery into cells. DNA Cell Biol. 21: 937-951.

Schwarz, D.S., Hutvagner, G., Du, T., Xu, Z., Aronin, N., and Zamore, P.D. 2003. Asymmetry in the assembly of the RNAi enzyme complex. Cell 115: 199-208.

Song, J.J., Smith, S.K., Hannon, G.J., and Joshua-Tor, L. 2004. Crystal structure of Argonaute and its implications for RISC slicer activity. Science 350: 1434-1437.

Sontheimer, E.J. and Carthew, R.W. 2004. Argonaute journeys into the heart of RISC. Science 305: 140-141.

Sui, G., Soohoo, C., Affar, B., Gay, F., Shi, Y., Forrester, W.C., and Shi, Y. 2002. A DNA vector-based RNAi technology to suppress gene expression in mammalian cells. Proc. Natl. Acad. Sci. 99: 5515-5520.

ter Brake, O. and Berkhout, B. 2007. Lentiviral vectors that carry anti-HIV shRNAs: Problems and solutions. J. Gene Med. 9: 743750.

Tiscornia, G., Singer, O., Ikawa, M., and Verma, I.M. 2003. A general method for gene knockdown in mice by using lentiviral vectors expressing small interfering RNA. Proc. Natl. Acad. Sci. 100: 18441848.

Tran, N., Cairns, M.J., Dawes, I.W., and Arndt, G.M. 2003. Expressing functional siRNAs in mammalian cells using convergent transcription. BMC Biotechnol. 3: 21. doi: 10.1186/1472-6750-3-21.

Vickers, T.A., Koo, S., Bennett, C.F., Crooke, S.T., Dean, N.M., and Baker, B.F. 2003. Efficient reduction of target RNAs by small interfering RNA and RNase $\mathrm{H}$-dependent antisense agents. A comparative analysis. J. Biol. Chem. 278: 7108-7118.

Waterhouse, P.M., Wang, M.B., and Lough, T. 2001. Gene silencing as an adaptive defence against viruses. Nature 411: 834-842. 
Westerhout, E.M. and Berkhout, B. 2007. A systematic analysis of the effect of target RNA structure on RNA interference. Nucleic Acids Res. 35: 4322-4330.

Wunsche, W. and Sczakiel, G. 2005. The activity of siRNA in mammalian cells is related to the kinetics of siRNA-target recognition in vitro: Mechanistic implications. J. Mol. Biol. 345: 203-209.

Yang, J.P., Fan, W., Rogers, C., Chatterton, J.E., Bliesath, J., Liu, G., Ke, N., Wang, C.-Y., Rhoades, K., Wong-Staal, F., et al. 2006. A novel RNAi library based on partially randomized consensus sequences of nuclear receptors: Identifying the receptors involved in amyloid beta degradation. Genomics 88: 282-292.

Yee, J.K. 1999. Retroviral vectors. In The development of human gene therapy (ed. T. Friedmann), pp. 21-45. Cold Spring Harbor Laboratory Press, Cold Spring Harbor, NY.
Zhang, J., Wang, C., Ke, N., Bliesath, J., Chionis, J., He, Q.S., Li, Q.X., Chatterton, J.E., Wong-Staal, F., and Zhou, D. 2007. A more efficient RNAi inducible system for tight regulation of gene expression in mammalian cells and xenograft animals. RNA 13: 1375-1383.

Zheng, L., Liu, J., Batalov, S., Zhou, D., Orth, A., Ding, S., and Schultz, P.G. 2004. An approach to genomewide screens of expressed small interfering RNAs in mammalian cells. Proc. Natl. Acad. Sci. 101: 135-140.

Zhou, D., He, Q.S., Wang, C., Zhang, J., and Wong-Staal, F. 2006. RNA interference and potential applications. Curr. Top. Med. Chem. 6: 901-911.

Zhou, D., Wang, C., Zhang, J., Bliesath, J., He, Q.S., Ke, N., Yu, D., Li, Q., Zhang, L., and Wong-Staal, F. 2008. Generation of shRNA pool library: A revision of the biological technique from the viewpoint of chemistry. ChemBioChem 9: 1365-1367. 

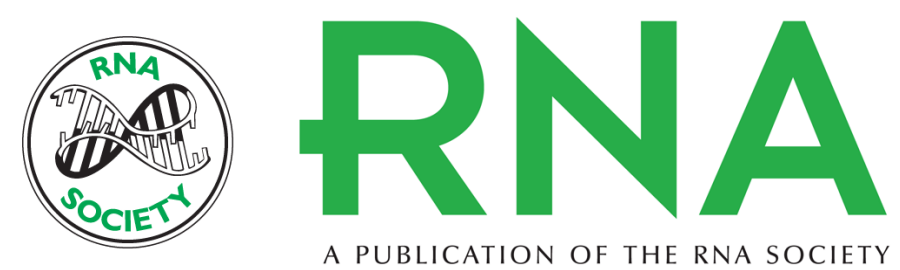

\section{A method for detecting and preventing negative RNA interference in preparation of lentiviral vectors for siRNA delivery}

Demin Zhou, Jing Zhang, Cuiying Wang, et al.

RNA 2009 15: 732-740 originally published online February 25, 2009

Access the most recent version at doi:10.1261/rna.985209

\section{References This article cites 44 articles, 13 of which can be accessed free at: http://rnajournal.cshlp.org/content/15/4/732.full.html\#ref-list-1}

License Email Alerting $\begin{aligned} & \text { Receive free email alerts when new articles cite this article - sign up in the box at the } \\ & \text { Service }\end{aligned}$ top right corner of the article or click here. 